\title{
The Laplacian energy of conjugacy class graph of some dihedral groups
}

\author{
Rabiha Mahmoud a, Amira Fadina Ahmad Fadzil a, Nor Haniza Sarmin a, ${ }^{*}$, Ahmad Erfanian b \\ ${ }^{a}$ Department of Mathematical Sciences, Faculty of Science, Universiti Teknologi Malaysia, 81310 Skudai, Johor, Malaysia \\ ${ }^{b}$ Department of Mathematics and Center of Excellence in Analysis on Algebraic Structures, Ferdowsi University of Mashhad, Mashhad, Iran \\ *Corresponding author: nhs@utm.my
}

\section{Article history}

Received 28 February 2017

Accepted 28 March 2017

\begin{abstract}
Let $G$ be a dihedral group and $\Gamma_{G}^{c l}$ its conjugacy class graph. The Laplacian energy of the graph, $L E\left(\Gamma_{G}^{c l}\right)$ is defined as the sum of the absolute values of the difference between the Laplacian eigenvalues and the ratio of twice the edges number divided by the number of vertices. In this research, the Laplacian matrices of the conjugacy class graph of some dihedral groups and its eigenvalues are first computed. Then, the Laplacian energy of this graph is determined.
\end{abstract}

Keywords: Dihedral groups, conjugacy class graph, Laplacian energy, Laplacian matrix, eigenvalues

\section{INTRODUCTION}

A graph $\Gamma$ is a finite nonempty set of objects called vertices together with a set of unordered pairs of distinct vertices of $\Gamma$ called the edges (Chartrand et al., 2010). The vertex-set of $\Gamma$ is denoted by $V(\Gamma)$, while the edge-set is denoted by $E(\Gamma)$.

Let $\Gamma$ be a graph with set of vertices $V(\Gamma)=\{1, \ldots, n\}$ and the set of edges $E(\Gamma)=\left\{e_{1}, \ldots, e_{n}\right\}$. The adjacency matrix of $\Gamma$ denoted by $A(\Gamma)$, is an $n \times n$ matrix defined as follows: the rows and the columns of $A(\Gamma)$ are indexed by $V(\Gamma)$. If $i \neq j$, then the $(i, j)$-entry of $A(\Gamma)$ is 0 for nonadjacent and 1 for adjacent vertices $i$ and $j$. The $(i, i)$-entry of $A(\Gamma)$ is 0 for $i=\{1, \ldots, n\}$ (Beineke and Wilson, 2004). The degree of vertex $i$ is denoted by $d_{\Gamma}(i)$ and the degree matrix on the other hand denoted by $D(\Gamma)$ is defined as $D(\Gamma)=\operatorname{diag}\left(d_{\Gamma}(1), d_{\Gamma}(2), \ldots, d_{\Gamma}(n)\right)$ which is the diagonal matrix of vertex degrees. Then, the Laplacian matrix is denoted by $L(\Gamma)$ hence is defined as $L(\Gamma)=D(\Gamma)-A(\Gamma)$ (Teranishi, 2011). Then, the Laplacian energy of the graph $\Gamma$ is defined as the sum of the absolute values of the difference between the Laplacian eigenvalues and the ratio of twice the edges number divided by the vertices number (Gutman and Zhou, 2006). By the Laplacian eigenvalues of the graph we mean the eigenvalues of its Laplacian matrix.

Suppose $G$ is a finite group. Two elements $a$ and $b$ of $G$ are said to be conjugate if there exists an element $g \in G$ with $g_{a g}{ }^{-1}=b$ (Rotman, 1995). The conjugacy class of a group $G$ is an equivalence relation and therefore partition $G$ into some equivalence classes. This means that every element of the group $G$ belongs to precisely one conjugacy class. The equivalence class that contains the element $a \in$ $G$ is $\operatorname{cl}(a)=\left\{g a g^{-1}: g \in G\right\}$ and is called the conjugacy class of $a$ (Rotman, 1995). The classes $\operatorname{cl}(a)$ and $\operatorname{cl}(b)$ are equal if and only if $a$ and $b$ are conjugate. The class number of $G$ is the number of distinct (non equivalent) conjugacy classes and we denote it by $K(G)$. In this paper we introduced the laplacian energy of conjugacy class graph of dihedral groups.

\section{PRELIMINARIES}

In this section some concepts on the conjugacy class graph which are used in the following section are presented.

\section{Definition 1 (Bertram, et al., 1990)}

Let $G$ be a finite group and let $Z(G)$ be the center of $G$. The vertices of conjugacy class graph of $G$ are non-central conjugacy classes of $G$ i.e. $V(G)=K(G)-|Z(G)|$, where $K(G)$ is the class number of $G$. Two vertices are adjacent if their cardinalities are not coprime (i.e. have common factor).

\section{Theorem 1 (Samaila, et al., 2013)}

The conjugacy classes in dihedral group $D_{2 n}$ are as follows, depending on the parity of $n$.

1. For odd $n$ :

$$
\{1\},\left\{a, a^{-1}\right\},\left\{a^{\frac{n}{2}}\right\} \ldots,\left\{a^{\frac{n-1}{2}}, a^{-\left(\frac{n-1}{2}\right)}\right\},\left\{a^{i} b, 0 \leq i \leq n-1\right\} .
$$

2. For even $n$ :

$$
\begin{aligned}
& \{1\},\left\{a, a^{-1}\right\},\left\{a^{2}, a^{-2}\right\}, \ldots,\left\{a^{\frac{n-2}{2}}, a^{-\left(\frac{n-2}{2}\right)}\right\},\left\{a^{2 i} b, 0 \leq i \leq \frac{n-2}{2}\right\} \text { and } \\
& \left\{a^{2 i+1} b, 0 \leq i \leq \frac{n-2}{2}\right\} .
\end{aligned}
$$

Proposition 1 (Mahmoud, et al., 2016)

Let $D_{2 n}$ be a dihedral group of order $2 n$ where $n \geq 3, n \in \mathbb{Z}^{+}$. Then the conjugacy class graphs of $D_{2 n}$, are as follows: 
Case 1: For $n$ odd : $\Gamma_{D_{2 n}}^{c l}$ is a complete graph joined with isolated vertices, namely $\Gamma_{D_{2 n}}^{c l}=K_{\frac{n-1}{2}}$.

Case 2: For $n$ and $\frac{n}{2}$ even : $\Gamma_{D_{2 n}}^{c l}=K_{\frac{n+2}{2}}$.

Case 3: For $n$ even and $\frac{n}{2}$ odd $: \Gamma_{D_{2 n}}^{c l}=K_{\frac{n+2}{2}} \cup K_{2}$.

Proposition 2 (Beineke and Wilson, 2004)

The multiplicity of 0 as eigenvalue of $L(\Gamma)$ is equal to the number of connected components of the graph.

Proposition 3 (Bapat, 2010)

The Laplacian matrix of the complete graph $K_{n}$ has eigenvalues 0 with multiplicity 1 and $n$ with multiplicity $n-1$.

\section{MAIN RESULTS}

In this section we present our main results, namely the Laplacian energy of the conjugacy class graph of some dihedral groups of order $2 n, n \geq 3, n \in \mathbb{Z}^{+}$. First we found the eigenvalues, the number of edges and the number of vertices of $\Gamma_{D_{2 n}}^{c l}$.

\section{Proposition 4}

Let $D_{2 n}=<a, b \mid a^{n}=b^{2}=1, b a b^{-1}=a^{-1}>$ be a dihedral group of order $2 n$, where $n \geq 3, n \in \mathbb{Z}^{+}$and $\Gamma_{D_{2 n}}^{c l}$ be its conjugacy class graph . The Laplacian eigenvalues of $\Gamma_{D_{2 n}}^{c l}$ are $\mu=0$ with multiplicity 2 and $\mu=\frac{n-1}{2}$ with multiplicity $\frac{n+3}{2}$.

\section{Proof}

Consider $G=D_{2 n}$ is a dihedral group of order $2 n$, where $n$ is an odd integer and $n>3$ by using Proposition $1 \Gamma_{D_{2 n}}^{c l}$ is a complete graph $K_{n-1}$ joined with one isolated vertex, hence by using Proposition 2 the $^{2}$ multiplicity of 0 as Laplacian eigenvalue of $\Gamma_{D_{2 n}}^{c l}$ is equal to $\overline{2}$ and by using Proposition 3 the Laplacian eigenvalues of $K_{\frac{n-1}{2}}$ is ${ }_{2}^{n-1}$ with multiplicity $\frac{n-1}{2}-1=\frac{n-3}{2}$, that gives $\mu=\frac{n-1}{2}$ with multiplicity $\begin{gathered}n-3 \\ 2\end{gathered}$.

\section{Proposition 5}

The number of the vertices of the conjugacy class graph of dihedral group $D_{2 n}=<a, b \mid a^{n}=b^{2}=1, b a b^{-1}=a^{-1}>$ where $n$ is an odd integer and $n>3$ is $\left|V\left(\Gamma_{D_{2 n}}^{c l}\right)\right|=\frac{n+1}{2}$.

\section{Proof}

Consider $G=D_{2 n}$ is a dihedral group of order $2 n$, where $n$ is an odd integer and $n>3$ by using proposition $1, \Gamma_{D_{2 n}}^{c l}$ is a complete graph $K_{n-1}$ joined with one isolated vertex, hence the number of vertices of $\Gamma_{D_{2 n}}^{c l}{ }^{2}$ is equal to $\frac{n-1}{2}+1=\frac{n+1}{2}$.

\section{Proposition 6}

Let $D_{2 n}=<a, b \mid a^{n}=b^{2}=1, b a b^{-1}=a^{-1}>$ be a dihedral group of order $2 n$, where $n \geq 3, n \in \mathbb{Z}^{+}$and $\Gamma_{D_{2 n}}^{c l}$ be its conjugacy class graph. The number of edges of $\Gamma_{D_{2 n}}^{c l}$ is: $\left|E\left(\Gamma_{D_{2 n}}^{c l}\right)\right|=\frac{n^{2}-4 n+3}{8}$.

\section{Proof}

Consider $G=D_{2 n}$ is a dihedral group of order $2 n$, by using Proposition $1 \Gamma_{D_{2 n}}^{c l}$ is a complete graph $K_{\frac{n-1}{2}}$ joined with one isolated vertex, from Proposition 3 the number of edges of a complete graph $K_{n}$ is equal to $\frac{n(n-1)}{2}$, hence the number of edges of $\frac{K_{n-1}}{2}$ is $\left|E\left(\begin{array}{c}K n-1 \\ 2\end{array}\right)\right|=$ $\frac{\left(\frac{n-1}{2}\right)\left(\frac{n-1}{2}-1\right)}{2}=\frac{n^{2}-4 n+3}{8}$.

\section{Proposition 7}

Let $D_{6}=<a, b \mid a^{3}=b^{2}=1, b a b^{-1}=a^{-1}>$ be the dihedral group of order 6 . Then the Laplacian energy of the conjugacy class graph of $D_{6}$, $L E\left(\Gamma_{D_{6}}^{c l}\right)=0$.

\section{Proof}

Consider $D_{6}=\left\{1, a, a^{2}, b, a b, a^{2} b\right\}$, then the conjugacy classes of $D_{6}$ are $\operatorname{cl}(1)=\{1\}, \operatorname{cl}(a)=\left\{a, a^{2}\right\}$ and $\operatorname{cl}(b)=\left\{b, a b, a^{2} b\right\}$. This gives $V\left(\Gamma_{D_{6}}^{c l}\right)=\{c l(a), \operatorname{cl}(b)\}$ and $\operatorname{gcd}(|c l(a)|,|c l(b)|=1$, hence by the definition of the conjugacy class graph, $\Gamma_{D_{6}}^{c l}$ is an empty graph and $L E\left(\Gamma_{D_{6}}^{c l}\right)=0$

\section{Theorem 2}

Let $D_{2 n}=<a, b \mid a^{n}=b^{2}=1, b a b^{-1}=a^{-1}>$ be a dihedral group of order $2 n$, where $n$ is an odd integer, $n \geq 3, n \in \mathbb{Z}^{+}$and $\Gamma_{D_{2 n}}^{c l}$ be its conjugacy class graph. Then the Laplacian energy of $\Gamma_{D_{2 n}}^{c l}, L E\left(\Gamma_{D_{2 n}}^{c l}\right)=$ $\frac{2\left(n^{2}-4 n+3\right)}{n+1}$

\section{Proof}

By the definition of the Laplacian energy we have $L E\left(\Gamma_{G}\right)=$ $\sum_{i=1}^{n}\left|\mu_{i}-\frac{2 m}{n}\right|$, where $\mu_{i}$ are Laplacian eigenvalues of $\Gamma_{G}, m$ is the number of edges and $n$ is the number of vertices. From Proposition 4 , Proposition 5 and Proposition 6 the Laplacian energy of $\Gamma_{D_{2 n}}^{c l}$ is given as in the following:

$L E\left(\Gamma_{D_{2 n}}^{c l}\right)=2\left|0-2\left(\frac{\frac{n^{2}-4 n+3}{8}}{\frac{n+1}{2}}\right)\right|+\left(\frac{n-3}{2}\right)\left|\left(\frac{n-1}{2}\right)-\left(\frac{\frac{n^{2}-4 n+3}{8}}{\frac{n+1}{2}}\right)\right|$

Hence, $L E\left(\Gamma_{D_{2 n}}^{c l}\right)=\frac{2\left(n^{2}-4 n+3\right)}{n+1}$.

We illustrate the above theorem with the following example.

\section{Example 1}

Let $D_{10}=<a, b \mid a^{5}=b^{2}=1, b a b^{-1}=a^{-1}>$ be a dihedral group of order 10. We have $\Gamma_{D_{2 n}}^{c l}=K_{2}$ joined with isolated vertex, hence $L\left(\Gamma_{D_{10}}^{c l}\right)=\left[\begin{array}{ccc}1 & -1 & 0 \\ -1 & 1 & 0 \\ 0 & 0 & 0\end{array}\right]$, and the characteristic equation of $L\left(\Gamma_{D_{10}}^{c l}\right)$ is $\operatorname{det}(\mu I-L)=\mu^{3}-2 \mu^{2}=0$. This gives the eigenvalues $\mu=0$ with multiplicity 2 and $\mu=2$ with multiplicity 1 . Hence, the Laplacian energy of the conjugacy class graph of $D_{10}$,

$$
L E\left(\Gamma_{D_{10}}^{c l}\right)=2\left|0-\frac{2}{3}\right|+\left|2-\frac{2}{3}\right|=\frac{8}{3} .
$$

\section{CONCLUSION}

In this paper, the general formula for the Laplacian energy of conjugacy class graph of dihedral groups $D_{2 n}$ is found when $n$ is an odd integer and $n>3$.

\section{ACKNOWLEDGEMENT}

The authors would like to acknowledge UTM for the Research University Fund (GUP) for vote no. 13H79 and 11J96 and the first author would like to appreciate UTM for partial financial support through International Doctorate Fellowship (IDF).

\section{REFERENCES}

Bapat, R. B. 2010. Graphs and Matrices. New York: Springer. Beineke, L. W., Wilson, R. J. 2004. Topics in algebraic graph theory (Volume 102). New York: Cambridge University Press. 
Bertram, E. A., Herzog, M., Mann, A. 1990. On a graph related to conjugacy classes of groups. Bulletin of the London Mathematical Society. 22(6), $569-575$.

Chartrand, G., Lesniak, L., Zhang, P. 2010. Graphs \& digraphs (Volume 39). Chemical Rubber Company (CRC) Press.

Gutman, I., Zhou, B. 2006. Laplacian energy of graph. Linear Algebra and Its Applications. 414(1), 29-37.

Mahmoud R., Sasrmin N., H., Erfanian A. 2016. The energy of conjugacy class graph of dihedral groups. International Graduate Conference on
Engineering, Science and Humanities, Universiti Teknologi Malaysia, UTM Press. 315-318.

Rotman, J., J. 1995. An Introduction to the Theory of Groups. 4th ed. New York: Spring Verlag.

Samaila, D., Abba, B. I., Pur, M. P. 2013. On the conjugacy classes, centers and representation of the groups $S_{n}$ and $D_{n}$. International Journal of Pure and Applied Sciences and Technology, 15(1), 87-89.

Teranishi, Y. 2011. Subgraphs and the Laplacian spectrum of graph. Linear Algebra and Its Applications. 435(5), 1029-1033. 